\title{
DESIGN AND DEVELOPMENT OF A FLEXIBLE WORKFLOW SUPPLY CHAIN SYSTEM
}

\author{
H.C.W. Lau \\ Dept. of Manufacturing Engineering \\ The Hong Kong Polytechnic University, Hong Kong \\ Email: mfhenry@inet.polyu.edu.hk \\ A. Ning \\ Dept. of Manufacturing Engineering \\ The Hong Kong Polytechnic University, Hong Kong \\ Email: mfandrew@inet.polyu.edu.hk \\ F.T.S. Chan \\ Dept. of Industrial \& Manufacturing System Engineering \\ University of Hong Kong, Hong Kong \\ Email: ftschan@hkucc.hku.hk \\ R.W.L. Ip \\ Dept. of Manufacturing Engineering \& Engineering Management \\ City University of Hong Kong, Hong Kong \\ Email: mewlraip@cityu.edu.hk
}

\begin{abstract}
This paper attempts to suggest a methodology for the design and development of a Flexible Workflow Supply Chain (FWSC) system for achieving flexibility to cope with unexpected changes. In particular, a multi-agent approach, which provides the capability to meet the requirement of a FWSC system, is proposed. Such system is characterized by its capability to cope with the increasing complexity setting of organizations and markets, providing effective information exchange between business partners. The methodology for the design and development of a workflow agent is also covered in this paper. The significance of contribution of this paper is concerned with the suggestion of a multiagent methodology, which is able to enhance the effectiveness in terms of the information flow in a supply chain, thereby providing more alternatives and ideas for those researchers who are interested in this field of study.
\end{abstract}

Keywords: Supply Chain, Multi-Agent, Workflow Design, Workflow Modeling

\section{INTRODUCTION}

In general, supply chain is a set of activities that span enterprise functions from ordering and receipt of raw materials through the manufacturing of products through the distribution and delivery to the customers. By flattening out their organizations and making use of ad-hoc workgroups or project teams, customer-focused business activities can become more amenable to ever-changing market conditions. Apart from the issue related to customers, the role of information flow is equally important to construct an effective supply chain system, linking partner companies with distinct competence on a global basis.

From another point of view, workflow is related to the movement of business activities within a supply chain. In general, business activity can be typically defined as an independent piece of work that can be performed by one or more persons in one time interval. Evidences $[1,2]$ have been shown that the adoption of workflow can help regulate and monitor the flow of information around the company. By managing these activities more efficiently, a company can acquire tangible as well as intangible savings in terms of its products and services, thereby giving itself a vital edge over its competitors. As a result, in order to stay responsive towards market condition, flexibility is an important issue that needs to be given more consideration in the formulation of a company's workflow $[3,4]$.

These requirements make supply chain an obvious application area for adopting multi-agent technology. This is because problem solving in the multi-agent approach is performed by means of communication between a set of loosely coupled independent agents. 
Such agents are business-oriented, and they try to accomplish business goals in complex ever-changing markets $[5,6]$.

A Flexible Workflow Supply Chain (FWSC) system, which is equipped with the compatibility to cope with the increasing complexity setting of organizations and markets, is proposed in this paper. The proposed FWSC system is going to support the business partners in the supply chain to extract and transport data effectively and efficiently, so that companies can use the existing information to support diverse strategies for design, manufacturing and distribution. The framework for the implementation of the proposed FWSC system incorporates the Object Request Broker (ORB) technology in order to improve the information flow within the system [7, 8].

The design and development of a workflow agent include the procedures of design as well as modeling. The design phase is very much related to the formulation and analysis of the existing movement of the business activities within a company. The modeling phase aims to develop a model for a reconfigurable system. In short, the creation of a workflow agent helps to unify the previously disparate business divisions that serve different regional operations. Finally, the proposed FWSC system lends itself to exploit the underlying concern of the organization, such as product and process quality, customer satisfaction and general business objectives.

\section{FLEXIBLE WORKFLOW SUPPLY CHAIN (FWSC) SYSTEM}

In this research, the main focus is the business activities relating to enterprise planning in strategic level and enterprise scheduling strategies in operational level in a supply chain [9]. Since multiagent co-ordination cannot be accomplished in the same level, hence the proposed FWSC system framework is divided into two parts shown in Figure 1.

In the strategic level, the workflow agent consists of five co-ordination agents, namely, Order Acquisition agent, Scheduling agent, Transportation Management agent, Resource Management agent and Logistics agent. These agents are based on corresponding functions performed in traditional supply chain. Concurrently, they aim to develop preliminary business activity plans and scheduling strategies prior to delivering to the operational level transaction agents for subsequent actions.

Each transaction agent makes particular plans and scheduling strategies based on its resource capacity information that is collected from business partners. Then each transaction agent sends the corresponding task to the business partners. At the same time, information systems in the business partners interact with the FWSC system for updating information. Thus, the workflow agent executes the particular plans and scheduling strategies that are received from each transaction agent.

A Data Communication Translator (DCT) is adopted for communication among the agents. Data interchanges between the co-ordination agents and system repository are via ORB, in order to enable efficient data exchange among various information systems from business partners that may reside in distributed platforms over geographically-isolated regions.

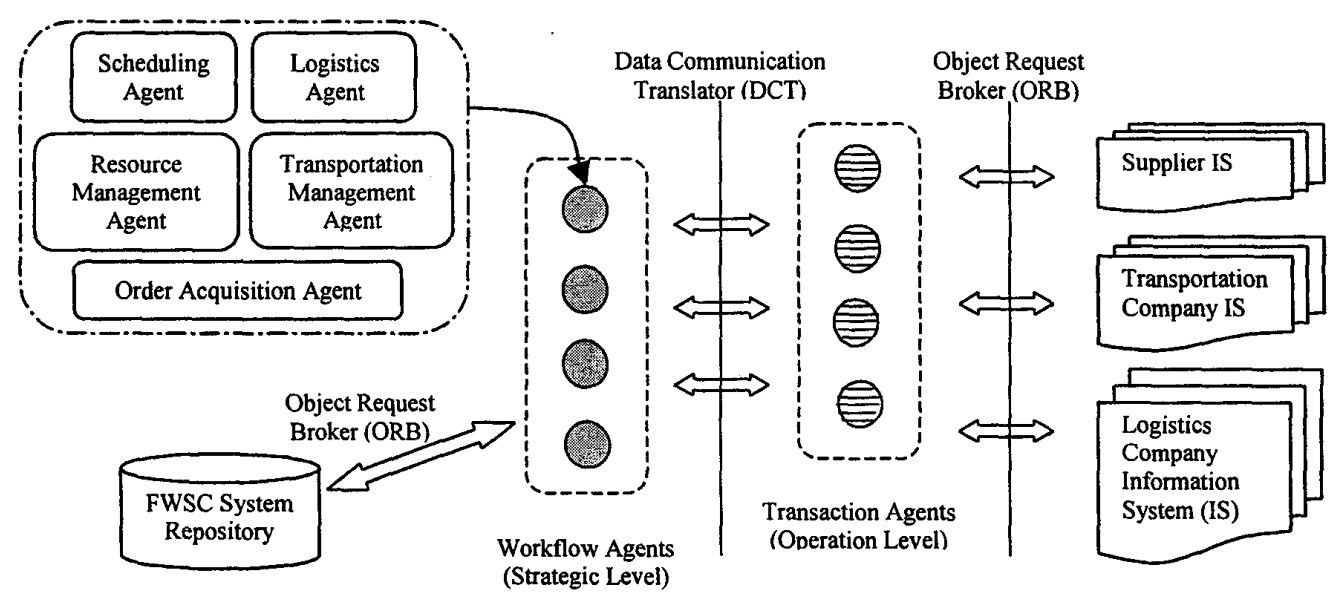

Figure 1: Multi-Agent Co-ordination Framework of Flexible Workflow Supply Chain (FWSC) System 


\section{WORKFLOW AGENT DESIGN}

The design of a workflow agent is very much related to the formulation and analysis of the existing business activities, such as product design and process planning. The focal point is to identify and name the existing business activities within the system. A good methodology to identify business activities has been presented by Hammer [10]. Such methodology covers listing current business activities and expresses initial and final states of those activities, as illustrated in Table 1.

Having identified the business activities, it is essential to outline the elements that formulate that particular activity. In general a business activity can be conceptualized via people, information and task. For instance, the 'Product Design' activity can be analytically decomposed into the following elements, such as customer and product designer (human); customer requirement and design specification (information); customer requirement analysis and design authorization (task). With the completion of the design, it creates a pool of information, which is going to be applied later in the modeling phase.

Table 1: Identifying and naming some examples of business activities

\begin{tabular}{|l|l|}
\hline Business Activity & Initial and Final states \\
\hline Product Design & $\begin{array}{l}\text { Customer requirement } \\
\text { analysis to design } \\
\text { authorization }\end{array}$ \\
\hline Process Planning & $\begin{array}{l}\text { Process identification to } \\
\text { scheduling }\end{array}$ \\
\hline Logistics & Planning to delivery \\
\hline
\end{tabular}

\section{WORKFLOW AGENT MODELING}

In the modeling phase, the focus is on the modeling of a flexible system infrastructure. Firstly, the company needs to form project teams from each division to develop visual business activity models. Then before attempting to develop a workflow agent model of a company, it should be noted that system analysts and developers, as well as end users should have understood some fundamental principles of modeling. (E.g. The modeling process is a collaborative process and it should have addressed all dimensions of business activities.)

Next, with the assistance of modeling tools, the project teams can develop workflow agent models that visually depict the existing business activities in each division. Although there are numerous modeling tools available, it should be noted that all of them are based on some kind of the standards, hence it is essential to learn the fundamental principles of those standards. In this research, an 'object' modeling techniques called Unified Modeling Language (UML) [11] has been employed due to its neutral and universally-accepted standards in creating all the relevant schemas [12]. The benefits of the development of workflow agent models help each company divisions understand their current business activities, thereby gaining insight into what the other divisions are doing.

\section{THE INFORMATION FLOW IN FWSC SYSTEM}

The information exchange among the business partners and the frequency of information updating have a strong influence on the efficiency of the supply chain [13]. With the emergence of the need to do business globally, any extension of a supply chain means more information has to be exchanged between business partners' information systems. However, each business partner may have a different kind of information system. Consequently, the information that has been created is very heterogeneous, and it is very difficult to assemble this kind of information into an application.

In this research, in order to make the proposed FWSC system as interoperable as possible, the ORB technology has been incorporated for increasing the degree of integration so as to provide more usefiul features to the proposed systems. The ORB can be regarded as a common interface over which requests are passed between objects (data). The purpose of an ORB is to ensure interoperability between applications on different machines in a heterogeneous distributed environment. Common Object Request Broker Architecture (CORBA), which is built around an ORB, can be regarded as a standard architecture for distributing objects in networks that aiming to simplify the communication between the heterogeneous information.

Through CORBA standard, the organization of data can be unified so that diversified applications can exchange relevant data. Normally, companies can only analyze their own internal data assets and reports from market before formulating their own strategies. However, incorrect data may also have the adverse effect of causing a wrong strategic direction. So the prospect of giving every business partners a total and unified view of the present situation would bring numerous benefits (such as better resource planning), thus improving companies' competitiveness. In short, the CORBA standard provides flexibility on the company's investment (a company can make the right choice for its particular environment and needs), improve the manageability on the company (more 
effective information handling on business activities and relationships) and enhance the speed on application development (better communication between functional departments).

\section{TESTING AND EVALUATION}

Based on the proposed FWSC system framework, a prototype system has been developed. A testing and evaluation plan, which aims to determine the feasibility of such system in a real industrial situation, has been depicted as follows.

I. The first phase involves the technical evaluation of the design and operations of the system prototype, by verifying that the subroutine programs are doing the job as specified in the FWSC system framework.

II. The second phase is concerned with system evaluation. The prototype, with modifications made according to the problems encountered in the first phase, is deployed in a local area network, and the results are observed and recorded by the project team members.

III. The third phase is concerned with the site evaluation of the system. It is important that the FWSC system be field-tested by the real end-users, in order to determine the possible problems when operating in a practical environment.

At the present stage, the first phase has been completed, and phase two will commence once the modification of the system prototype and the setting up of a local area network have been finalized.

\section{CONCLUSION}

To compete in today's business environment, companies are required to integrate business activities into a global response strategy. To cope with sudden changes, companies need to conduct their business activities in a flexible manner. In this paper, the design and development of a FWSC system, which is characterized with its capability to achieve efficient information exchange in various value chain activities of the logistics distribution network, is proposed. For the implementation of the proposed FWSC system, companies should take a look at their whole business activities and identify areas for revolutionary or evolutionary changes. They can take advantage of technology developments to build alternative forms of communication and partnership with supply and distribution partners. The result, if handled effectively, is essential to provide insights related to the strategic use of the supply chain concept, thereby significantly enhancing the competitiveness of companies in the ever-changing marketplace.

\section{ACKNOWLEDGEMENT}

The authors wish to thank the Department of Manufacturing Engineering of the Hong Kong Polytechnic University for the support of this research project.

\section{REFERENCES}

[1] P. Lawrence (Editor), Workflow handbook 1997, John Wiley \& Son, Chichester, 1997.

[2] G. Poyssick and S. Hannaford, Workflow Reengineering, Adobe Press, Mountain View, California, 1996.

[3] S. Ellis, K. Keddara and G. Rozenberg, "Dynamic Change within Workflow Systems", presented at the ACM Conference on Organizational Computing Systems (COCS 95), 1995.

[4] G. Kappel, P. Lang, S. Rausch-Schott and W. Retschitzegger, "Workflow Management Based on Object, Rules and Roles", IEEE Data Engineering, March 1995.

[5] K. Sycara, K. Decker, A. Pannu, M. Williamson and D. Zeng, "Distributed Intelligent Agents", IEEE Expert, December 1996.

[6] H. Nwana, "Software Agents: An Overview", Knowledge Engineering Review, vol. 11, no. 3, pp. 205-244, October/November 1996.

[7] A. Pope, The CORBA Reference Guide: Understanding the Common Object Request Broker Architecture, Addison-Wesley Longman Limited, Essex, 1998.

[8] Object Management Group (OMG), CORBA/IIOP Specification, http://www.omg.org, 2000.

[9] H.L. Lee and C. Billington, "The Evolution of Supply Chain Management Models and Practice at Hewlett-Packard", Interface, vol. 25, no. 5, September/October 1995.

[10] M. Hammer, Reengineering the corporation: a manifesto for business revolution, Harper Business, New York, 1993. 
[11] J.B. Warmer, The object constraint language: precise modeling with UML, Addison Wesley, Longman, 1999.

[12] Jacobson, The Object Advantage - Business Process Reengineering with Object Technology, ACM Press Books, 1995.

[13] ADC News \& Solutions (magazine articles), "Driving the supply chain with better information", in News and Application Trends of the ADC News \& Solutions online, May, 1998, http://www.manufacturing.net/magazine/adc/. 